tions which must be present in order to insure the permanent checking of the malady.

I have alluded above to the fact that attention to the general health offers the best chances of helping sclerosis in all its forms, and that on this account more is to be hoped from the use of change of air, cod-liver oil, malt extract, and the mechanical tonics, such as massage and electricity, than from drugs supposed to have a more specific value.

Another reason for the false encouragement to be had at times out of the clinical history of scleroses of the cord, and especially out of locomotor ataxia, is the fact that besides the definite sclerosis, with the equally definite mischiefs it causes, there is another coincident element of trouble which varies. This is probably a congestive state, and may with reason be supposed to exist in and about the sclerotic patches, and perhaps elsewhere. I think this element of ataxic cases has been quite too much overlooked, but to it we must turn for an explanation of the sudden increase or lessening of symptoms, and to the false appearance of gain in many cases of posterior sclerosis. I have over and over seen cases in which there was for a time complete ataxy of both arms and hands, so that no useful motion was possible, and I have seen this condition almost entirely disappear for months or even years. In one gentleman, now in my charge, this loss and gain has taken place twice, and each time there has been left a larger residuum of permanent mischief.

Whatever be the varying element of trouble in posterior sclerosis it is influenced largely by exercise, which, whenever it is extreme, is apt to be hurtful to such cases. This is why some ataxics are always best on rising from the night's sleep, and why, especially in the earlier stages of ataxia, long periods of absolute rest are so useful. I have already described elsewhere the influence of repose on some of the phenomena of this disease, and it is perhaps enough, here, to remind you that $I$ have over and over seen the neuralgia of this disease pass away permanently, or for long periods, owing to rest in bed for two months.

\title{
A CASE OF INVERSION OF THE UTERUS OF NINE MONTHS' STANDING. ${ }^{1}$
}

BY E. H. stevens, M. D., CAMBRidge.

Mrs. C., aged twenty-two years, French Canadian, was first seen by me September 18, 1876, when she presented the following history: She menstruated for the first time at the age of thirteen years, and continued regular and in robust health. She married at the age of twenty. Eight months afterwards she became pregnant, and went to full term. She first began to have pains January 18, 1876. The pains

1 Read before the Boston Society for Medical Observation, October 7, 1878. 
continued five days and nights, being severe enough to prevent sleep. January 23d she was delivered of a large female child, the second stage of labor lasting sixteen hours. A physician was constantly in attendance during this time. Owing to the protracted nature of the labor, the harassing pains, and the loss of sleep, she had become much exhausted. Two hours after the birth of the child, the placenta not being delivered, the physician passed his hand into the uterus and removed it, at the same time stating to the family that "it had grown to the side." Very profuse hæmorrhage followed, with loss of consciousness for a short time. Severe after-pains occurred throughout the day, accompanied by spells of fainting. A constant oozing of blood continued, notwithstanding the free use of ice and other remedies. The pains subsided in twenty-four hours, but the flowing continued in a more than normal quantity for three days, being at times severe. At the end of that time the flowing became less, and the patient was comfortable, suffering only from weakness. A good supply of milk was secreted, and she was able to nurse her child. Three weeks after confinement she got up to have her bed made, when flowing again set in, lasting with more or less severity for about three weeks. After this time there was but little discharge, and she began to gain strength. She sat up with her wrapper on when her baby was ten weeks old, and was very soon able to be about the house and attend to her domestic duties. From that time up to the present she has had an almost continuous sanguineous discharge, to control which various kinds of treatment, both local and general, have been tested by different physicians familiar with the case. One had applied cotton saturated with a preparation of iron, which controlled the hæmorrhage for five weeks. Another, supposing the trouble to be falling of the womb, introduced a rubber ring-pessary. This she was wearing when $I$ first saw her. Although able to be about the house and nurse her infant, her face and lips were of a chalky whiteness. Her appetite was good. At times she had trouble in seeing, and dizziness. A distinct murmur was noticed over the apex of the heart, which was increased on slight exertion. On making a vaginal examination, a tumor as large as a small orange was found low down in the vagina, and resting on the perinæum, easily movable in all directions. It could be freely handled without causing pain, the surface being rough and having a spongy feel. If scraped with the finger this roughness came off, leaving a smooth and shining mucous membrane underneath. After a somewhat careful and prolonged examination I satisfied myself that $I$ had to do with a case of inversion of the uterus.

The next day, September 19th, Drs. J. L. Hildreth and H. E. Marion saw the case in consultation, and verified my diagnosis. She being at once put under the influence of ether, and laid upon her back, with the 
knees drawn up, the diagnosis was made absolutely certain by passing the right hand into the vagina and pressing up the entire mass, with the left hand pressing down from above the pubes. In this manner the absence of the fundus of the uterus could be easily made out. Instead of the fundus of the uterus a distinct ring could be felt, into which the finger could be pressed, and the outlines of the broad ligaments could be made out. An attempt at reduction was now begun in the following manner: The uterus was grasped between the thumb and finger, the fundus resting in the palm of the hand; firm pressure was made from within the vagina, while the left hand steadied and supported the uterus from above the pubes. The fingers of the hand within the vagina were used as a wedge to dilate the cervix, after the plan described by Dr. Emmet in a case published by him in 1868, in the January number of the American Journal of the Medical Sciences, page 91.

After one hour and three quarters the cervical canal was sufficiently dilated to allow the fundus to be pushed very nearly within the uterine cavity. The state of the patient was such that it was deemed wise to discontinue any further attempt at reduction at this time. The state of the organ had entirely changed; from being hard and unyielding, it had become soft and pliable, and apparently not more than two thirds as large. To secure what had been gained, a rubber air-bag was applied, the fundus being pushed up as far as possible. Reaction from the ether came on slowly, but in twelve hours the patient's condition was about the same as before the operation. The air-bag was kept in position sixty hours, being removed twice, and an injection of warm carbolized water used. In reapplying the air-bag care was taken to have the fundus pushed well up into the uterine canal. At the end of sixty hours ether was again administered, the patient being in a fair condition, the pulse being 120 per minute. Drs. Hildreth, Marion, and W. W. Dow were present, and rendered valuable assistance. On removing the air-bag the uterus at once dropped into the vagina, but could be pushed into the canal again, so that the condition seemed about the same as when the last operation was abandoned. For two hours I continued my efforts to reduce the organ when the fundus was entirely within the canal, the cervix coming together over it. It appeared to all present that complete reduction would be accomplished in a very short time. I was, however, obliged to -desist on account of the patient's condition, she having a pulse of 160 , with difficult respiration. Not wanting to lose anything we had gained, I determined to adopt Emmet's plan of passing sutures through the cervix, and thus keep the fundus within the uterine canal. Four deep, interrupted silver-wire sutures were passed through the neck of the uterus and twisted, bringing the sides of the cervix together. In two hours the patient had reacted from her ether, and was left comfortable. One hour and a half after leav- 
ing the house a messenger came for me in great haste, saying that the woman was dying. I reached her bedside in a short time, to find her a little faint, but otherwise comfortable. The nurse reported that about one hour before very severe pains came on in the lower part of the abdomen, extending through to the back. They were described as like labor pains, but much more severe. After lasting three quarters of an hour something was felt to give way inside, when all pain suddenly ceased, leaving only faintness behind. This soon passed away, and she said that she had not felt so well before for months. The pulse was good. There was tenderness above the pubes. One eighth of a grain of morphine was ordered every two hours, with hot fomentations. At my next visit, six hours afterwards, the pulse was found to be good; micturition somewhat difficult; less tenderness than at last visit; no tympanites. She had vomited several times, and felt sleepy, but could not sleep. Morphine was administered less frequently, and fomentations were continued. On visiting her the next morning she was reported to have passed a comfortable night, getting several hours' quiet sleep; pulse less than 100 , temperature $99^{\circ} \mathrm{F}$.; very little tenderness over any part of the abdomen. She said that she felt well enough to get up. No vomiting since the previous evening. The stomach retained beef tea and milk punch. She had to wait some time before she could pass water. Morphine was discontinued. Not feeling sure that the uterus had replaced itself, although all the symptoms would warrant that supposition, I thought it best to wait until the next day before removing the stitches, when I could have assistance. Accordingly, on the morning of the 22d, Drs. Marion and Dow being present, ether was again administered, and the stitches were removed, when we found that the organ had entirely regained its normal position, the fundus being plainly felt from above the pubes. The cavity of the uterus, as measured by the Simpson sound, showed a depth of three and one quarter inches. From this time all hæmorrhage stopped, and she gained steadily. At the end of six days she was sitting up, and in ten days walked out, contrary to my advice. The following February menstruation appeared naturally. The family soon after moved to a distant part of the State. I learned by a letter from the husband, dated February 10, 1878, that his wife had a fine boy, born in January, and that she was in perfect health.

\section{A NEW MICROTOME. 1}

BY 8. W. FLETCher, M. D., PEPPEReLL, Mass.

Several years ago, wishing to make some thin sections of animal tissue, and not having the educated hand, I set about devising an instrument for doing such work. The conditions to be fulfilled appeared to

1 Shown to the Boston Society of Medical Sciences, November 30, 1878. 